\title{
Técnica ETL para informar a los comerciantes sobre los productos agrícolas comercializados en las plazas de mercado en la ciudad de Bogotá
}

\section{ETL technique to inform traders about agricultural products, marketed in marketplaces in the city of Bogotá}

Gabriel Alexis Roncancio Hernández

Fundación Universitaria Uninpahu - Correo electrónico:groncanciohe@uninpahu.edu.co

Laura Katherine Velosa Arias

Fundación Universitaria Uninpahu - Correo electrónico: Ivelosaar@uninpahu.edu.co -
Rubén Darío Angulo Quemba Fundación Universitaria Uninpahu - Correo electrónico: ranguloqu@uninpahu.edu.co-

Carlos Gilberto Donoso

Fundación Universitaria Uninpahu - Correo electrónico: cdonosoal@uninpahu.edu.co 


\section{INTRODUCCIÓN}

Un hecho que afecta hoy en día a las plazas de mercado es la fluctuación de los precios en los productos agrícolas. Esto genera en algunos casos un aumento notable en la variación de los precios de venta al consumidor, viéndose afectado tanto el usuario como el comerciante. El primero debido a que en muchos casos no puede adquirir el producto; y el segundo porque no obtiene ninguna ganancia durante el día.

Para responder efectivamente a esta situación los autores de este trabajo crearon una herramienta tecnológica con características de flexibilidad, usabilidad, portabilidad y confiabilidad. En su consecución, siguieron un proceso y unas metodologías que responden a los requerimientos y necesidades de la población objeto de investigación.

Esta herramienta busca como resultado final un producto con estándares de calidad que cubra las necesidades anteriormente mencionadas. La base con la que se construyó el modelo proviene de referencias y análisis estadísticos, de donde surgieron los datos y la información necesaria para la construcción del producto.

Para desarrollar la herramienta fue necesario tener en cuenta la Inteligencia de Negocios o BI (por sus siglas en inglés), que es la habilidad para transformar los datos en información de conocimiento, de forma que se pueda optimizar el proceso de toma de decisiones en los negocios [1].

Conscientes de la importancia del BI para la creación de la herramienta tecnológica, se generó una investigación referente a la fuente, que permitiera extraer los datos necesarios para alimentar una base de datos pertinente, y además que la fuente de los datos fuera confiabley actuali- zada periódicamente. La fuente principal de información fue la página de la central mayorista de Corabastos, la cual es de dominio público y se utilizó en conjunto con el proceso de extracción, transformación y carga de datos (ETL). Luego se generaron los indicadores y estadísticas, que se ponen a disposición de los comerciantes en pro del beneficio de las plazas de mercado.

Este proyecto se orientó a las Pymes (Pequeñas y medianas empresas) y a todos los comercializadores de productos agrícolas interesados en herramientas que sirvan de soporte para la compra y comercialización de un producto.

Con el estudio, que se realizó por medio de encuestas y entrevistas, se evidenció que existe la necesidad e interés de los comerciantes de mejorar y optimizar los procesos en los que participan por medio de tecnologías de la información. Cabe decir que según las estadísticas:

en promedio, el 80\% de las Pymes fracasa antes de los cinco años y el $90 \%$ de ellas no llega a los 10 años. Para los dueños de Pymes, las razones del fracaso es necesario buscarlas fuera de las empresas, pero los analistas empresariales se orientan más a identificar las causas del fracaso en las propias Pymes y, en particular, en la capacidad de gestión de sus responsables [2].

Pero, en contraste con lo antes mencionado, en las encuestas realizadas en diferentes plazas de mercado de la ciudad de Bogotá, se encontró e que la población se encuentra interesada en herramientas que permitan estar al tanto de las tendencias y las variaciones de los precios en el momento de la compra y venta de los productos agrícolas.

Por lo anterior, se creó la herramienta ya antes descrita con la finalidad de que cualquier persona que emplee la herra- 
mienta pueda usar la información para el análisis, la reflexión y una adecuada toma de decisiones.

De acuerdo con todo lo planteado hasta ahora, los objetivos del presente artículo son: primero mostrar los resultados de la investigación y segundo, con base en lo anterior, dar a conocer la forma como se creó la herramienta tecnológica, basándose en estadísticas que proporcionen información de manera rápida y precisa con relación a los precios de los productos que se comercializan en las diferentes plazas de mercado.

Por otra parte, se define el proceso de extracción, transformación y carga de datos (ETL), y la importancia que ha tenido para la alimentación de la bodega de datos de la herramienta desarrollada. Además, se buscó generar informes periódicos con los precios y estimaciones de los productos de las plazas de mercado $\mathrm{y}$, a través de interfaces gráficas con características de usabilidad y portabilidad, se buscó la forma de mostrar la información los destinatarios inmediatos. Por último, una vez transcurrido un periodo de tiempo, la herramienta generó estadísticas basadas en los resultados recolectados y procesados para, de esa forma, generar tendencias en la variación de los precios de un producto.

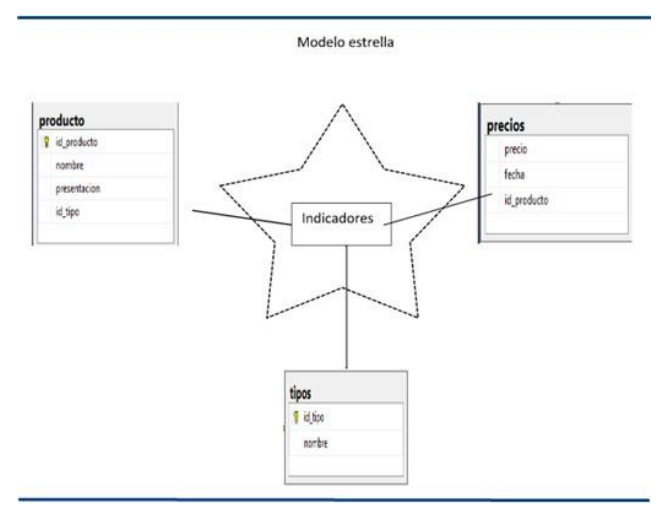

Figura 1. Modelo Estrella de la Bodega de Datos Fuente: Elaboración propia.

\section{METODOLOGÍA}

A continuación se precisa el tipo de estudio, las fuentes de información y las técnicas de recolección de información y definición de la muestra para la aplicación de pruebas:

Tipo de investigación

La investigación realizada fue de carácter explicativo y cuantitativo, expresando las causas de la problemática relacionada con la desinformación que enfrentan los comerciantes de las plazas de mercado. Se realizó un análisis de la situación actual del problema planteado, buscando una estrategia de ETL (Extracción, transformación y carga de datos) y BI (Inteligencia de negocios).

Luego se identificaron las principales herramientas para lograr los estándares óptimos de calidad que el presente proyecto requería. Se utilizaron 2 herramientas para la recolección de información (encuestas y entrevistas) del sector a tratar, que permitieron consolidar una idea más clara de la problemática.

El proyecto se desarrolló usando como caso de estudio la información que influía en el momento de la compra y venta de un producto en las plazas de mercado, y el proceso de obtención de la misma.

La obtención de las fuentes de información se clasificaron como:

\section{- Fuentes primarias:}

Información suministrada por los comerciantes de las plazas de mercado el Galán, Fontibón y Paloquemao principalmente, a partir de encuestas realizadas en el año 2016 orientadas al proceso de la compra del producto por parte de los comerciantes, y basados en que los comerciantes realizaban lo mencionado anteriormente.

\section{- $\quad$ Fuentes secundarias:}

Se realizó una búsqueda inicial a través del Internet y en diferentes bases de datos 
con el fin de conocer el estado del arte, y de esa manera detectar qué aportes innovadores podía brindar el presente proyecto.

Por otro lado se detallaron los tópicos y criterios de consultas e investigación relacionados con las plazas de mercado donde posteriormente se realizaron las encuestas: Características del comerciante, accesibilidad al Internet, herramientas existentes, plataformas disponibles y de fácil acceso.

Además, como fuente secundaria adicional se usó la información disponible en los siguientes sitios web:

- $\quad$ Página de la plaza de mercado de Corabastos (Central mayorista de abastecimiento de Bogotá):

http://www.corabastos.com.co/sitio/

- $\quad$ Página de la plaza de mercado de Paloquemao:

http://www.plazadepaloquemao.com/

- $\quad$ Página de la plaza de mercado del barrio Galán:

http://www.ipes.gov.co/index. php/19-plazas-de-mercado/148-plaza-de-mercado-distrital-trinidad-galan Fontibón:

ht tp://www.ipes.gov.co/index. php/19-plazas-de-mercado/318-historia-de-la-plaza-distrital-de-fontibon

Técnicas de recolección de información

Uno de los inconvenientes más grandes para la construcción de la herramienta tecnológica fue el de entender cómo se fijaban los precios de los productos en las plazas de mercado. En el estudio, basado en observaciones, entrevistas y encuestas, se determinó que los comerciantes tienen muchos criterios para la fijación del precio de un producto. Sin embargo, tales crite- rios son insuficientemente precisos y muy variables para cada caso en particular.

Una de esas variables es la negociación entre el vendedor y los compradores, motivo por el cual se debe tener en cuenta el instante en el que sucede la negociación y el poder de persuasión de cada individuo, que depende también de unos rangos de precios en donde se decide cual es el precio más adecuado para el producto durante el día. Otras variables que se tienen en cuenta son el origen, la calidad y el trabajo que se requiera para la poscosecha de dichos productos.

Más en concreto, la fijación de precios de las hortalizas en Colombia se realiza en la central de acopio de la capital "CORABASTOS", y se rigen principalmente por la oferta y demanda de los productos; sin embargo, en algunas ocasiones, se rigen por la conveniencia de unos pocos o por mafias que son muy difíciles de identificar. Esto último hace que los precios de muchos productos agrícolas sean baratos a costa de la pérdida que ocasiona en los campesinos colombianos.

Precisamente, para Acosta [3], uno de los aspectos principales a desarrollar en la producción agropecuaria consiste en establecer la "fijación de precios", aspecto clave para la toma de decisiones en las empresas agrícolas o agroindustrias. No obstante en los sistemas de economía campesina esto muchas veces se desconoce [4].

De hecho, los precios fijados en los mercados campesinos obedecen a las fluctuaciones del mercado mayor, que tiene como base de información dos valores: los precios de la central mayorista CORABASTOS en tanto precio base o mínimo y los precios de las tiendas del sector en donde se realiza cada mercado presencial; a partir de estos precios se fijan los de todos los productos que se comercializan 
en este sistema, incluidas las hortalizas frescas que se venden en los parques. En este cálculo, se desconocen los costos en los que incurre un productor de economía campesina, que no maneja grandes volúmenes para ser evaluado en el mismo nivel de los productores mayoristas (CORABASTOS), es por ello que este trabajo nace de la necesidad de los mercados campesinos y sus financiadores por mejorar el sistema de información que permita generar precios más justos para productores y compradores de mercados campesinos de Bogotá [3].

\section{RESULTADOS}

Por medio de la siguiente encuesta se recolectó información sobre la necesidad de contar con una herramienta web a partir del interés de la población para saber los precios de los productos agrícolas que se comercializan en las diferentes plazas de mercado de la ciudad de Bogotá, así como de conocer los requerimientos de los comerciantes y las necesidades del sector sobre la información.

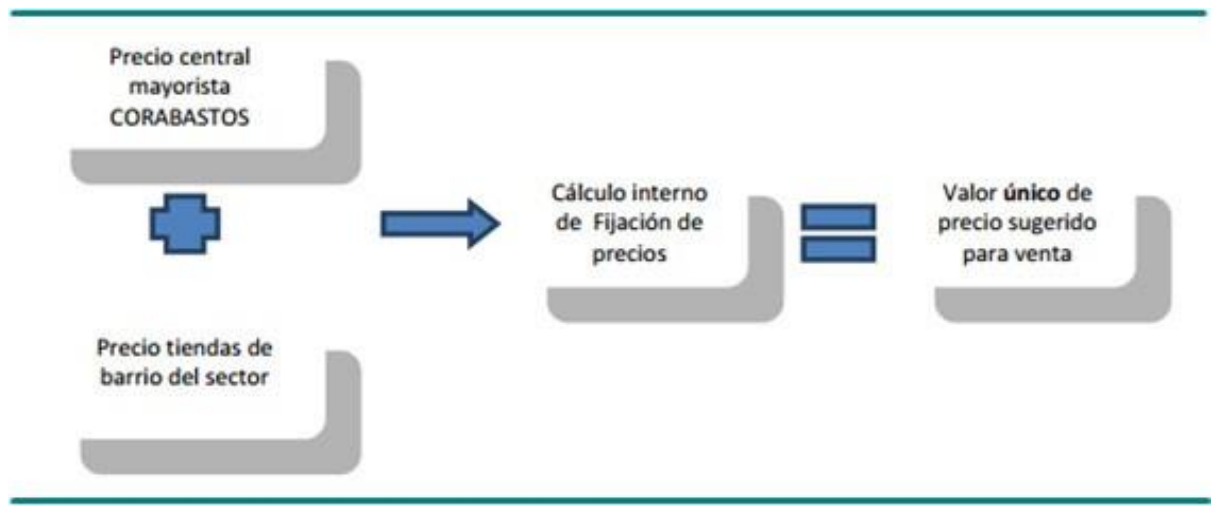

\section{Figura 1. Fijación de precios en mercados campesinos}

Fuente: [3]

Por otra parte, los mercados campesinos presenciales de Bogotá cuentan con otra gran dificultad en el tema de la fijación de precios y es que aun cuando el sistema debería ser de mercado intervenido, es decir, con la regulación de la organización, también se presenta un mercado libre, que se da cuando los productores tienen total libertad para decidir cuánto cobrar por sus productos y se presenta en consecuencia una libre negociación dependiendo de cuánto esté dispuesto a pagar el consumidor por ese producto [3].

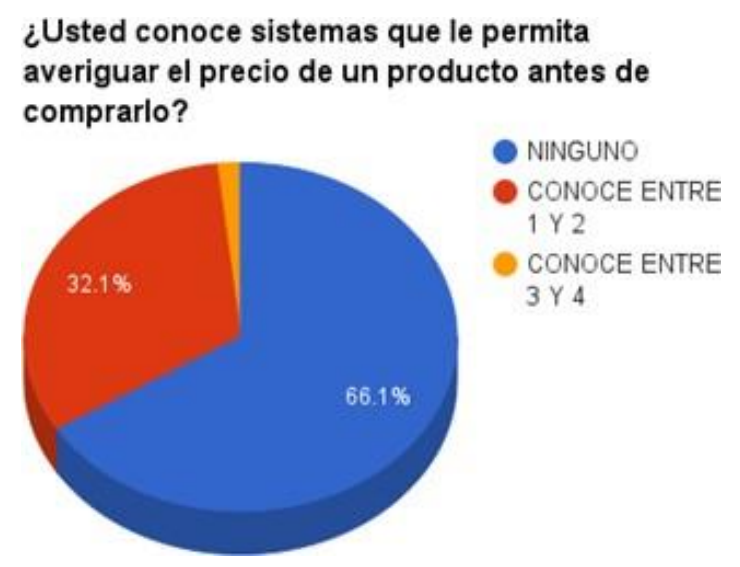

Figura 2. Conocimientos de los medios de comunicación

Fuente: Elaboración propia.

La encuesta evidencia que el $61.1 \%$ de la población encuestada no conoce he- 
rramientas informáticas, que le permita conocer los valores de los productos comercializados en las diferentes plazas, en tanto que el $32.1 \%$ conoce entre 1 a 2 medios de información (radio, televisión). Lo aanterior nos permite determinar que una gran parte de la población no permanece informada (Figura 1).

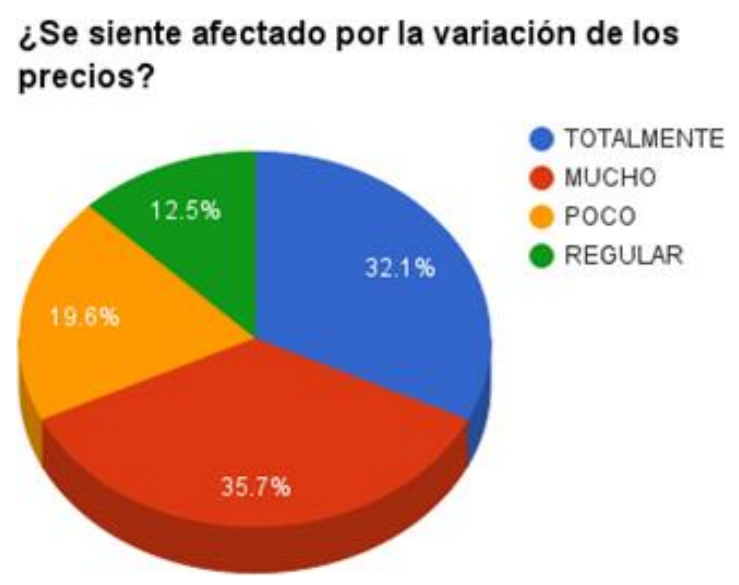

Figura 3. Afectación en variación de precios Fuente: Elaboración propia.

Por otro lado, se observó que el 67.8\% de la población se siente afectado por la variación de los precios tanto de compra como de venta (Figura 2).

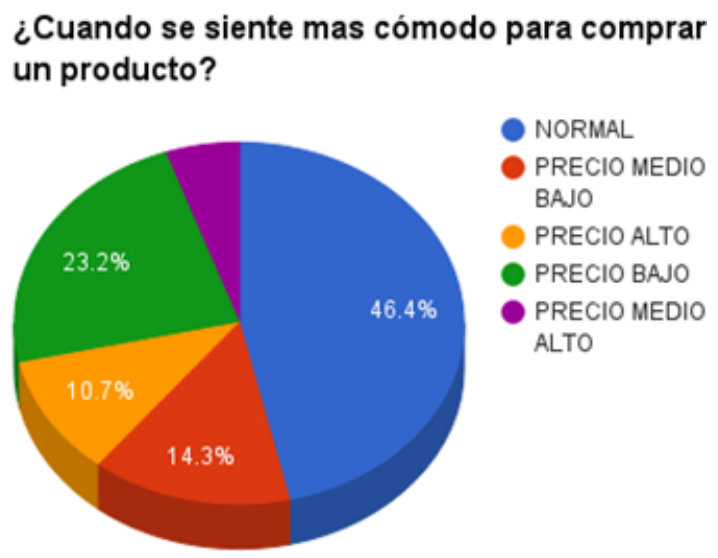

Figura 4. Compra de productos Fuente: Elaboración propia.
Esta misma afectación indica que los comerciantes prefieren comprar cuando los precios se encuentren estabilizados, tal como se puede observar en la Figura 4.

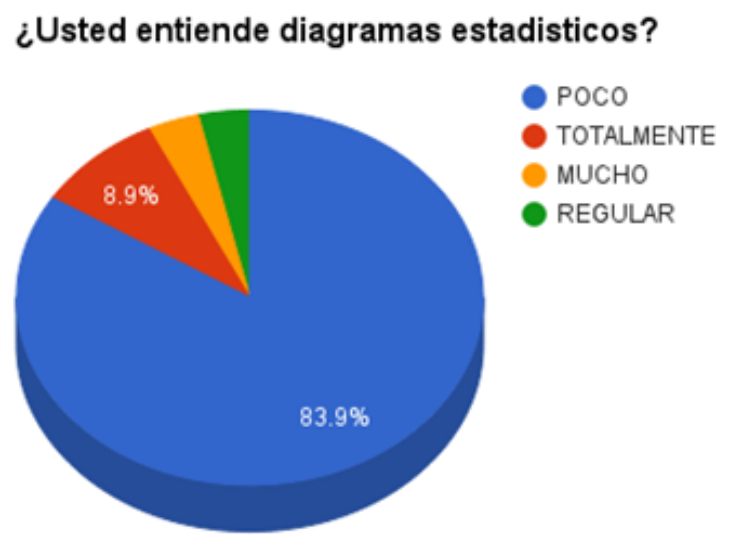

Figura 5. Comprensión de diagramas estadísticos

Fuente: Elaboración propia

En general, los comerciantes de la muestra no entienden diagramas estadísticos, debido a que no interactúan diariamente con ellos. Esta afirmación, dando un resultado de $83.9 \%$ de personas que no comprendía diagramas estadísticos (ver figura 5).

\section{¿Con que frecuencia busca información en la web?}

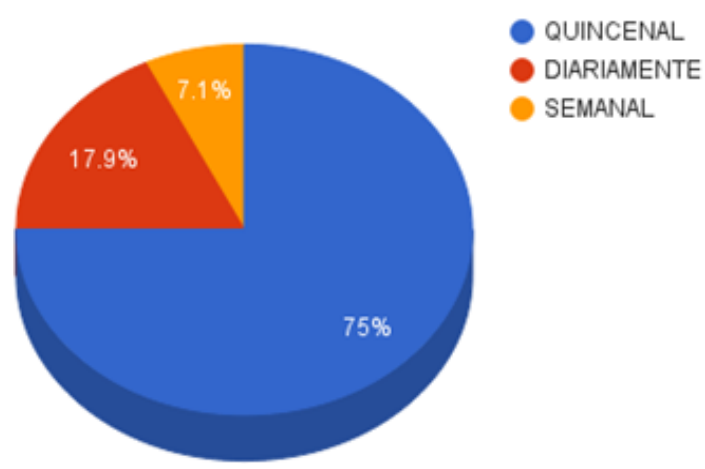

Figura 6. Buscar información Fuente: Elaboración propia. 
Unos de los datos preocupantes de la investigación fue el resultado acerca de la pregunta ¿Con qué frecuencia usted busca información en la web?, debido a que aun teniendo los medios tecnológicos los comerciantes no los utilizan para encontrar información relevante sobre su actividad comercial, no la están viendo como una solución (ver Figura 6).

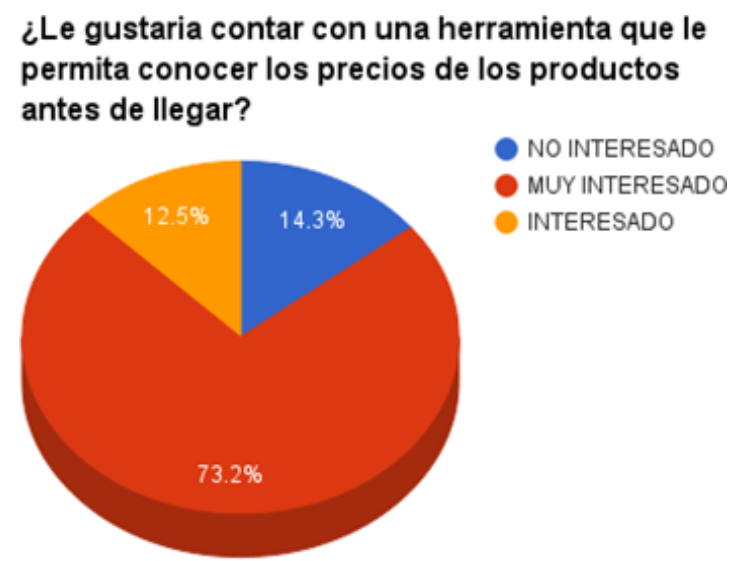

Figura 7. Interés por herramientas tecnológicas Fuente: Elaboración propia.

La encuesta informó que la mayoría de las personas encuestadas están interesadas en la creación de una herramienta web que le permitan acceder a la información desde cualquier lugar o momento, siendo este uno de los resultados más significativos, con un porcentaje de 73.2\% (ver Figura7).

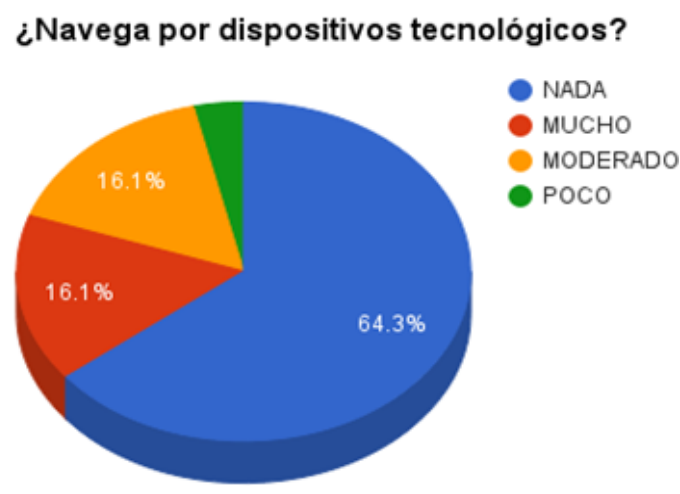

Figura 8. Frecuencia del uso de la tecnología Fuente: Elaboración propia
A pesar del interés por contar con una herramienta web, el $64.3 \%$ de los encuestados no navega por medios tecnológicos (Figura 8).

¿Por qué implementar una herramienta ETL es la solución adecuada?

Actualmente existen muchas herramientas, mecanismos y metodologías para la gestión de la información que permiten mejorar y facilitar la toma de decisiones, ampliar una visión con respecto a lo que sucede y contribuir en el desarrollo y calidad de vida del involucrado. En ello juega un papel importante la citada Inteligencia de Negocios (Business Intelligence-BI), que se define como El conjunto de técnicas, procesos y arquitectura que transforman los datos recopilados por una compañía en información importante y relevante para los procesos gerenciales, desde la disminución de costos, hasta la creación de nuevos negocios [5].

Además, la inteligencia de negocios aporta grandes beneficios:

- Incrementa sustancialmente la eficiencia, al permitir que el usuario acceda a la información de una manera rápida, logrando lo que quiere. Por otra parte, permite centralizar la información y visualizarla en un mismo lugar, que puede ser una plataforma, una herramienta, una aplicación, entre otras ahorrando tiempo de respuesta y permitiendo una toma de decisión más eficiente.

- Permite analizar tendencias y comportamientos en el mercado, tanto por parte del comerciante como del consumidor, y de esa manera convierte esos datos en información, que será procesada y consumida con el único propósito de generar una rentabilidad a mediano y largo plazo.

Componentes de la inteligencia de negocios (BI) 


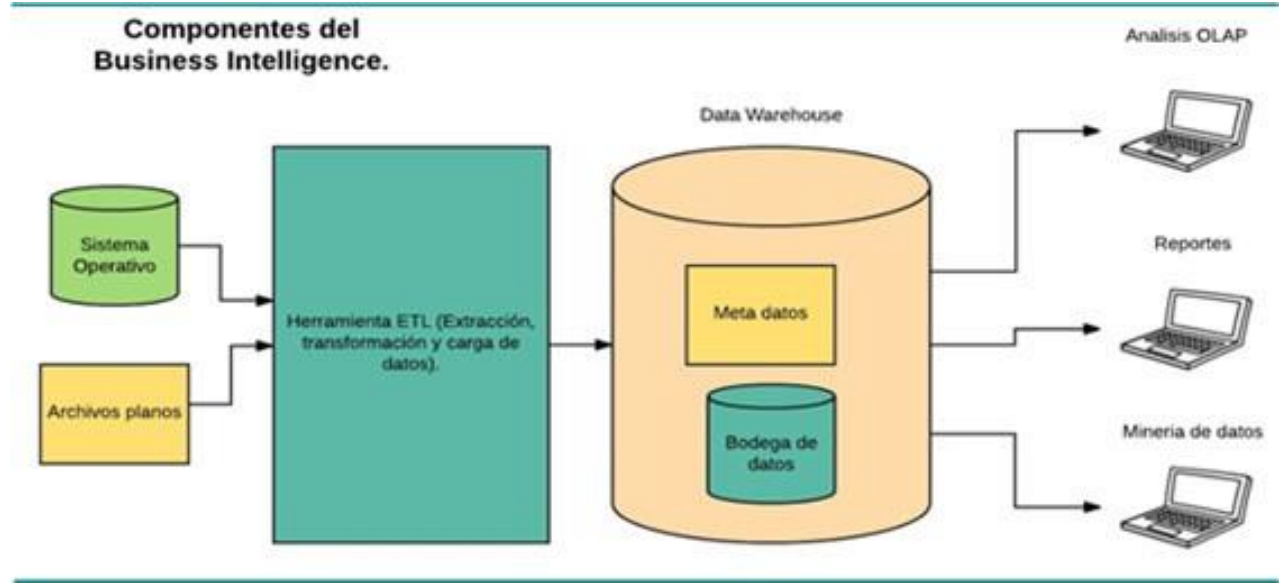

Figura 9. Componentes del Business Intelligence del presente proyecto. Fuente: Elaboración propia.

La gran mayoría de las veces, cuando se crean herramientas de inteligencia de negocios, se requiere crear, además, un esquema que permita visualizar la solución de BI. En Figura 4, se puede visualizar cómo está compuesto un sistema que integra una solución de BI. Así, en la parte derecha de la imagen se observa la identificación del lugar de donde se extraen los datos, que posteriormente son procesados por una herramienta ETL, para luego ser transformados y almacenados en una bodega de datos y terminar el ciclo, por medio de diferentes soluciones que permitan usar la información recolectada, bien sea a través de una solución de análisis de los datos OLAP, la generación de reportes o la minería de datos.

\section{DISCUSIÓN}

En este trabajo se encontró que la mayoría de los comerciantes de la plaza de mercados no encuentra la información necesaria por los medios tradicionales, y algunos tienen falencias de comunicación. La razón principal de esto radica en que las fuentes de información se limitan a la realización de llamadas a comerciantes de la misma plaza o de otras. De este modo, al no establecer la comunicación por diversos motivos, se genera una falla importante en la entrega de la información hacia el comerciante.

Por esta razón, se determinó buscar un medio por el cual los comerciantes no dependieran del "voz a voz" para determinar la compra de los productos agrícolas. Es paradójico que la mayoría de comerciantes tiene tecnologías de comunicación (computadores y dispositivos móviles), pero no la utilizan para encontrar la información en la web. Esto se debe a que gran parte de los comerciantes no conocen herramientas informáticas para esta función, y en la mayoría de casos solo utilizan la tecnología como medio de distracción. La herramienta web creada suple precisamente esta necesidad para los comerciantes, mostrando el precio de los productos agrícolas con actualizaciones diarias, además de un módulo para la creación de históricos e indicadores a fin de mostrar información de estadísticas. 
Así, por medio del big data y la inteligencia de negocios, los datos se transforman en información que luego puede ser mostrada a los comerciantes para el uso práctico en la rutina diaria.

\section{CONCLUSIONES}

La construcción de una herramienta web para informar a los comerciantes sobre los precios de los productos sirve de soporte a la perspectiva del comerciante, reduciendo la brecha de incertidumbre al momento de la compra de los productos. Con ello se logra una cercanía entre el comerciante y la información a través de una herramienta funcional, con portabilidad y usabilidad, que le permite mantenerse informado en todo momento. En la investigación se evidenció que la población afectada no implementa con eficiencia el uso de herramientas que permitieran notificar lo que sucede en el mercado con relación a los productos. Gracias a las estrategias de investigación, se detectaron las problemáticas por las cuales los encuestados no utilizan las tecnologías, y con base en lo anterior se construyó una herramienta web que cumple con las expectativas mínimas para que la población afectada la utilice diariamente.

- $\quad$ Por medio de la implementación de esta herramienta se pueden visualizar los precios de los productos y apoyar de este modo su actividad económica.

Por otro lado la herramienta destaca la inteligencia de negocios y las herramientas ETL para la extracción, transformación y carga de los datos, permitiendo pasar de datos a información relevante para los comerciantes.

Según sea la necesidad que tenga el comerciante, la herramienta permite tener una mayor panorámica y observar desde una óptica diferente el mercado, con el fin de llevar un mejor control y abonar en el crecimiento de los negocios de las plazas de mercado.

- $\quad$ Además, esta investigación permitió conocer en mayor profundidad el concepto de ETL, los orígenes, las técnicas y las diferentes herramientas con el propósito de llevar el conocimiento adquirido a la práctica, en un caso real y actual, pues, más que nunca es necesario implementar las TIC para el desarrollo y la competitividad de los negocios.

- $\quad$ Finalmente, la herramienta permitió que los comerciantes piensen en alternativas tecnológicas como mecanismos de apoyo para mejorar procesos.

En síntesis, la investigación plantea un mecanismo para que los comerciantes puedan interactuar con mayor frecuencia con las herramientas existentes, abriendo el camino hacia nuevos avances en la perspectiva del IB y a investigaciones posteriores.

\section{REFERENCIAS}

[1]. M. Garavito Triana, L. Gómez Soto, O. López Cadena, \& J. Valencia Camacho. "Diseño de herramienta de inteligencia de negocios para el manejo de la información de ventas de una empresa comercializadora de productos agropecuarios". 2015.

[2]. L. Soriano Claudio. GestioPolis, 2005. Disponible en: http://www.gestiopolis. com/por-que-fracasan-las-pymes/

[3]. D. A. Acosta Leal. "Fijación de precios en mercados campesinos de Bogotá. Caso hortalizas frescas de Fómeque y Chipaque (Cundinamarca)", 2014. Disponible en: http://www.bdigital.unal.edu. co/46578/1/710815.2014.pdf 
[4]. H. Mondragón y M. Flórez. La cuestión agraria hoy. Colombia: tierra sin campesinos. $1^{\text {a }}$ edición. ILSA. 2008.

[5]. P. Ortiz. (28 de 06 de 2013). "5 ventajas de la inteligencia de negocios". Revista M, Disponible en: http://mprende.co/ gesti \%C3\%B3n/5-ventajas-de-la-inteligencia-de-negocios

\section{Bibliografía de consulta}

- S. Vélez Quiroga. Herramienta para control y programación de la producción en el sector agrícola, 2011. Disponible en: http://www.bdigital.unal.edu. co/6958/1/822023.2011.pdf

- $\quad$ B. A. Narváez. Modelos de determinación de los precios agrícolas. Cuadernos de ciencias Económicas y empresariales. Núm. 11. España: Universidad de Málaga, 1983.

- Jonathan Nicolás Acosta Medellín y Daniel Humberto Flórez Lara (2015). Diseño e implementación de prototipo BI utilizando una herramienta de big data para empresas pymes distribuidoras de tecnología. Recuperado de http:/ / repository.ucatolica.edu.co/bitstream/10983/2543/1/PROYECTO\%20FINAL_.pdf y http:// repository.ucatolica. edu.co/handle/10983/2543

- $\quad$ A. Garrido, A. Gómez, J.E. Guerrero, V. Fernández.NIRS: una tecnología de apoyo para un servicio integral en alimentación animal, 1996. Disponible en: http:/ / www.acorex.es/es/pienso/NIRSunatecnologiadeapoyoparalaalimentacionanimal.pdf
- B. Solórzano, W. Pilligua Pincay y D. Noboa Macías.Diseño de un sistema de control de procesos empresariales basados en indicadores de gestión y desempeño para el proceso de ventas de una compañía dedicada a la comercialización de insumos agrícolas ubicada en la ciudad de Guayaquil para el año 2010. Disponible en: http://www.dspace.espol.edu.ec/ handle/123456789/16605 


\section{Este artículo se cita:}

G. Roncancio, R. Angulo, L. Angulo y C. Donoso, "Técnica ETL para informar a los comerciantes sobre los productos agrícolas comercializados en las plazas de mercado en la ciudad de Bogotá", Revista Investigación e Innovación en Ingenierias, vol. 5, n .1,pp.6071, 2017. 